\title{
Primary Liposarcoma of the Duodenum: A First Case Presentation
}

\author{
Takehiro Okabayashi ${ }^{1}$, Yasuo Shima ${ }^{1}$, Jun Iwata ${ }^{2}$, Tatsuaki Sumiyoshi ${ }^{1}$, Akihito Kozuki ${ }^{1}$, \\ Teppei Tokumaru ${ }^{1}$, Yasuhiro Hata ${ }^{3}$, Yoshihiro $\mathrm{Noda}^{3}$, Takeshi Inagaki ${ }^{2}$, \\ Saori Morishita ${ }^{4}$ and Masanori Morita ${ }^{4}$
}

\begin{abstract}
A 55-year-old man suffering from melena was admitted to our hospital. A blood test showed severe anemia. Contrast-enhanced computed tomography (CT) revealed a huge lesion in the duodenum and dilatation of the common bile duct. Upper gastrointestinal endoscopy also identified hemorrhaging from the tumor in the duodenum. Due to the low density of the tumor mass, we performed emergency pylorus-preserving pancreaticoduodenectomy. Histology revealed an area of well-differentiated liposarcoma as well as an area of highgrade spindle cells and pleomorphic sarcoma without obvious differentiation. The final pathological diagnosis was dedifferentiated liposarcoma. This is the first case report of primary liposarcoma of the duodenum.
\end{abstract}

Key words: liposarcoma, duodenum, CDK4, MDM2, pancreaticoduodenectomy

(Intern Med 52: 2753-2757, 2013)

(DOI: 10.2169/internalmedicine.52.1230)

\section{Introduction}

Liposarcoma is the most common soft tissue sarcoma and includes a number of subtypes that differ in their histological, biological and molecular features (1). This tumor often occurs in the thigh, behind the knee or in the retroperitoneal region of the abdomen, although it can also be found in other parts of the body (2-4). The World Health Organization recognizes five subtypes of liposarcoma: welldifferentiated, dedifferentiated, myxoid/round cell, pleomorphic and mixed-type. The myxoid/round cell subtype is among the most prevalent and typically occurs in younger individuals (5). Atypical lipomatous tumors/well-differentiated and dedifferentiated liposarcoma together form a larger subgroup of liposarcoma that is considered to represent a morphological and behavioral spectrum of the same disease (6).

Since surgical resection remains the only promising treatment for liposarcoma, performing total removal of the tumor with negative surgical margins is mandatory (7). Wide resection, including the neighboring organs, should be performed without hesitation in selected cases. The survival of patients with extremity tumors is favorable. However, the difficulty in obtaining wide margins in the retroperitoneum predisposes the patient to local recurrence and ultimately death from unresectable disease (8). We herein document the case of a patient suffering from primary liposarcoma of the duodenum with severe hemorrhage. To the best of our knowledge, this is the first report of primary liposarcoma of the duodenum. The patient underwent emergency pancreaticoduodenectomy with partial resection of the transverse colon. The surgery and postoperative course were uneventful, primarily due to the scrupulous preoperative evaluation.

\section{Case Report}

A 55-year-old man suffering from melena was referred to our hospital for an evaluation and treatment. He had no clinical evidence of any past malignancy. An examination

\footnotetext{
${ }^{1}$ Department of Gastroenterological Surgery, Kochi Health Sciences Center, Japan, ${ }^{2}$ Department of Diagnostic Pathology, Kochi Health Sciences Center, Japan, ${ }^{3}$ Department of Radiology, Kochi Health Sciences Center, Japan and ${ }^{4}$ Department of Gastroenterology and Hepatology, Kochi Health Sciences Center, Japan

Received for publication June 23, 2013; Accepted for publication July 29, 2013

Correspondence to Dr. Takehiro Okabayashi, takehiro_okabayashi@khsc.or.jp
} 


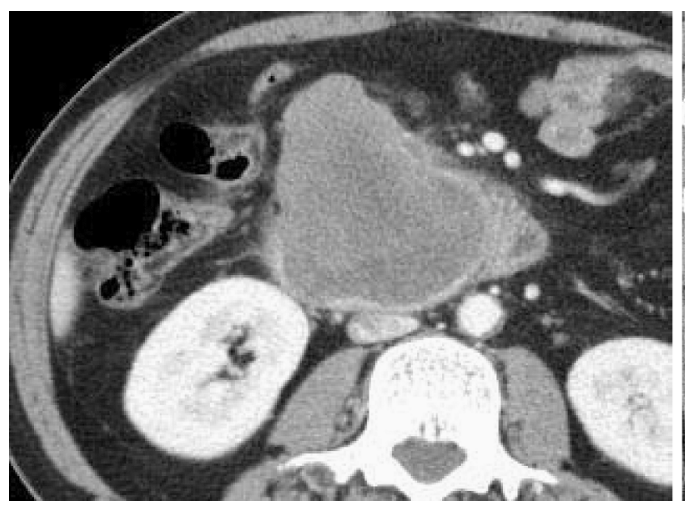

a

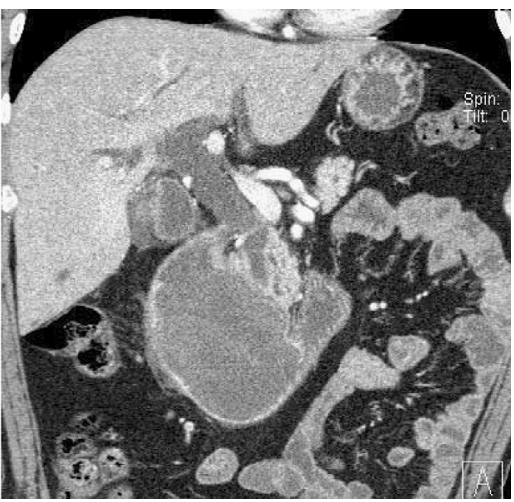

b

Figure 1. a: Abdominal contrast-enhanced CT revealed a large mass in the duodenum. b: CT also demonstrated both dilatation of the common bile duct and direct invasion into the transverse colon.

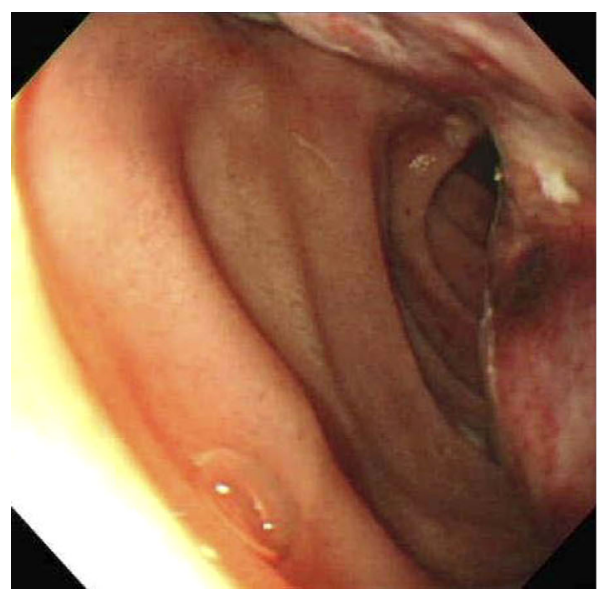

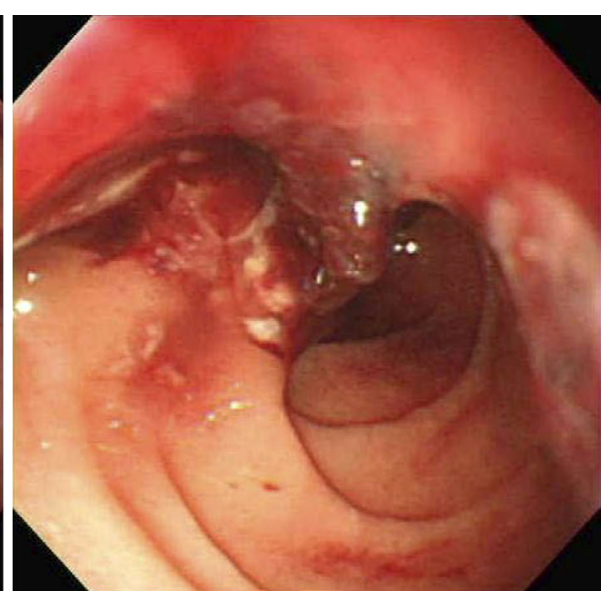

b

Figure 2. a: Upper gastrointestinal endoscopy identified hemorrhaging from the tumor in the duodenum. b: Endoscopic hemostatic procedures using clipping and ethanol injection failed to control the hemorrhage.

revealed a hard palpable mass in the abdomen and conjunctiva that were both icteric and markedly anemic. Laboratory blood test results demonstrated the following findings: $\mathrm{Hb}$, $6.7 \mathrm{~g} / \mathrm{dL}$ (normal: 13.4-17.4 g/dL); hematocrit, $21.2 \%$ (39.8$51.8 \%$ ); albumin, $2.6 \mathrm{~g} / \mathrm{dL}$ (3.8-5.3 g/dL); total bilirubin, 4.7 $\mathrm{mg} / \mathrm{dL}(0.2-1.2 \mathrm{mg} / \mathrm{dL})$; alkaline phosphatase (ALP), 3,636 IU/L (115-359 IU/L); and $\gamma$-glutamyl transpeptidase (GTP), $832 \mathrm{IU} / \mathrm{L}$ (16-73 IU/L). Other laboratory tests, including a blood smear, tumor marker analysis (carcinoembryonic antigen, carbohydrate 19-9 and carbohydrate 15-3) and serologic tests for viral infection, were within the normal ranges.

Abdominal contrast-enhanced computed tomography (CT) revealed a large and heterogeneous extraluminal mass measuring $9.5 \mathrm{~cm}$ in diameter arising from the second portion of the duodenum (Fig. 1a). CT demonstrated both dilatation of the common bile duct and direct invasion into the transverse colon (Fig. 1b). Upper gastrointestinal endoscopy identified hemorrhaging from the tumor, apparently originating from the duodenal submucosa; however, endoscopic hemostatic procedures using clipping and ethanol injection failed to control the hemorrhage (Fig. 2a, b). Due to the low density of the tumor mass, complete embolization of the arteries around the duodenum would potentially have had no effect and may have induced necrosis of the adjacent organs. The hemorrhage mass was diagnosed as a primary gastrointestinal stromal tumor based on the abdominal-enhanced CT findings, rather than a non-functioning endocrine tumor or leiomyosarcoma of the duodenum. Therefore, we performed emergency pylorus-preserving pancreaticoduodenectomy with partial resection of the transverse colon.

An evaluation of the resected specimen revealed a $9.8 \times$ 7.5-cm tumor in the duodenum (Fig. 3a). Macroscopically, the tumor was large, multilobular, well-circumscribed and appeared to be a white, solid, firm mass containing a yellow fatty area (Fig. 3b). Histologically, the tumor contained an area of well-differentiated liposarcoma in addition to an area of high-grade spindle cells and pleomorphic sarcoma without obvious differentiation (Fig. 3c). In the area of welldifferentiated liposarcoma, atypical spindle and stellate cells with large, irregularly shaped hyperchromatic nuclei were scattered in loose fibrous tissue and between mature-looking adipose cells (Fig. 3d). On the other hand, the area of high- 

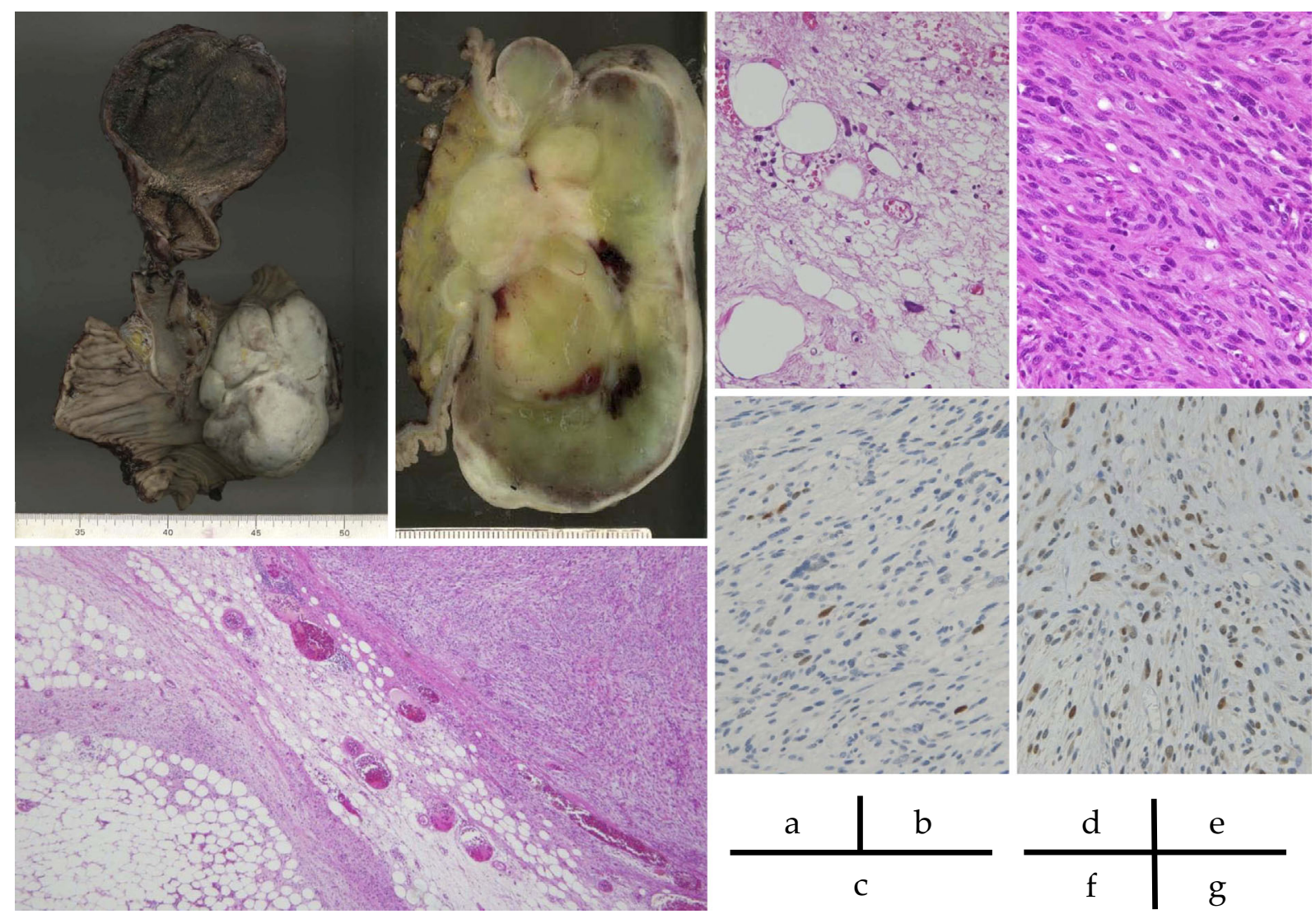

Figure 3. a: Evaluation of the resected specimen. b: Macroscopic findings. c: Gross appearance of the tumor. d: Well-differentiated liposarcoma. e: Area of high-grade spindle cells and pleomorphic sarcoma. f: Immunohistochemical staining for MDM2. g: Immunohistochemical staining for CDK4.

grade spindle cell and pleomorphic sarcoma, mimicking socalled malignant fibrous histiocytoma, exhibited a more cellular and atypical histology lacking obvious lipogenic differentiation (Fig. 3e). The tumor cells in the high-grade area showed positive immunostaining with antibodies to MDM2 (Fig. 3f) and CDK4 (Fig. 3g). The CDK4 staining was distributed diffusely, whereas MDM2-positive cells were scattered throughout the area and fewer in number than CDK4positive cells. The area of well-differentiated liposarcoma demonstrated the same immunohistochemical findings. The final pathological diagnosis was dedifferentiated liposarcoma. The patient's postoperative course was uneventful, and he was discharged four weeks after the surgery. Because liposarcoma was observed in the extremities and often metastasizes, the patient was followed by our department using systemic examinations, such as CT and magnetic resonance imaging, once every two months after discharge. There were no signs of recurrence of either the primary liposarcoma of the duodenum or gastrointestinal hemorrhage 10 months after emergency pancreaticoduodenectomy.

\section{Discussion}

We herein presented the first case report of primary liposarcoma arising in the duodenum that was successfully treated with emergency pylorus-preserving pancreaticoduo- denectomy.

Liposarcoma constitutes one of the most common soft tissue sarcoma groups in adults, of which well-differentiated liposarcoma and dedifferentiated liposarcoma comprise major subtypes. The histological diagnosis of adipocytic tumors has traditionally relied on morphology alone, with immunohistochemistry being of limited value; however, in recent years, the use of immunohistochemical staining for CDK4 and MDM2 has gained popularity, capitalizing on the distinct genetic background of well-differentiated/dedifferentiated liposarcoma compared with other adipocytic neoplasms (9-11). Like most immunohistochemical markers, MDM2 and CDK4 are not of absolute diagnostic value, and, as always, it is important to use these parameters as part of an immunostaining panel when approaching a particular case. However, in the appropriate clinical and pathological context, immunoreactivity for these markers can facilitate the accurate diagnosis of atypical lipomatous tumor/welldifferentiated liposarcoma and dedifferentiated liposarcoma. Ultimately, the primary duodenum tumor in this case was diagnosed as dedifferentiated liposarcoma, with typically scattered MDM2-positive nuclei, increased numbers of positive cells in the cellular areas and CDK4 reactivity that was stronger and more diffuse.

Surgical resection is the mainstay of curative treatment for patients with well-differentiated or dedifferentiated 
liposarcoma $(12,13)$. The present patient received emergency pylorus-preserving pancreaticoduodenectomy as a lifesaving procedure and the most appropriate management for primary liposarcoma of the duodenum with active hemorrhage. Subsequent gastrointestinal endoscopy detected the hemorrhage from the tumor; however, it was difficult to control the bleeding endoscopically. Recent reports suggest the usefulness of catheter intervention for treating gastrointestinal hemorrhage or periampullary neoplasms $(14,15)$. Due to the complex arterial anastomosis around the pancreatic head, the use of catheter intervention for tumors of the periampullary region is not sufficient. Complete embolization of the arteries around the duodenum may also be ineffective and was furthermore anticipated to induce necrosis of the adjacent organs in this case. Emergency pancreaticoduodenectomy is not a common procedure due to the associated rates of morbidity and mortality (16-19); however, recent advances in surgical techniques and improvements in perisurgical management have made it relatively safe, especially when performed by experienced surgeons in training centers.

New therapeutic modalities for treating liposarcoma, particularly aggressive tumors, including myxoid/round cell liposarcoma, are therefore needed. First, the expression of protein-coding genes in eukaryotes involves a number of tightly regulated steps, each of which is controlled by various proteins to ensure that the transcripts are appropriately expressed and processed. Protein translocation plays a significant role in the pathogenesis liposarcoma via the expression of a number of RNA polymerase II transcription genes, and the identification of several RNA polymerase II promoters in patients with liposarcoma has confirmed some target genes, including INTS3, RAS family, ZNF294 and $M E C P 2$ (20). The function of these target genes translocated in liposarcoma indicates their regulatory roles in processes as diverse as transcription, cell-cycle progression, DNA repair, genomic stability and neurodegeneration. Second, there has been a focus on identifying potential antigens for immunotherapy in patients with liposarcoma. NY-ESO-1 has been successfully targeted in trials of antigen-specific adoptive Tcell therapy, and complete responses have been observed in melanoma trials targeting NY-ESO-1 using both vaccines and adoptive transferred T-cells. Indeed, the discovery that synovial sarcoma expresses NY-ESO-1 was rapidly translated into a clinical trial (21). Furthermore, Pollack et al. (22) reported another soft tissue sarcoma subtype with a pattern of the NY-ESO-1 expression that is even more prevalent than that observed in synovial sarcoma. This latter study demonstrated that, like synovial sarcoma, myxoid/ round cell liposarcoma can be used as a model disease for the study of NY-ESO-1-directed therapy (22).

In conclusion, we herein reported the first case study of primary liposarcoma arising from the duodenum that was successfully treated with emergency pylorus-preserving pancreaticoduodenectomy for tumor bleeding that was not controlled with endoscopic intervention. This case suggests that pancreaticoduodenectomy can be selected as an appropriate treatment for aggressive hemorrhage from tumors of the pancreatic head as a life-saving procedure in specific situations.

The authors state that they have no Conflict of Interest (COI).

\section{Acknowledgement}

Supported by the Kochi Organization for Medical Reformation and Renewal Fund.

\section{References}

1. Gardner JM, Dandekar M, Thomas D, et al. Cutaneous and subcutaneous pleomorphic liposarcoma: a clinicopathologic study of 29 cases with evaluation of MDM2 gene amplification in 26. Am J Surg Pathol 36: 1047-1051, 2012.

2. Demetri GD, Chawla SP, von Mehren M, et al. Efficacy and safety of trabectedin in patients with advanced or metastatic liposarcoma or leiomyosarcoma after failure of prior anthracyclines and ifosfamide: results of a randomized phase II study of two different schedules. J Clin Oncol 27: 4188-4196, 2009.

3. Rosai J, Pilotti S. Retroperitoneal sarcoma. N Engl J Med 351: 105, 2004.

4. Lin YY, Su WF. Liposarcoma of the anterior mediastinum and neck. N Engl J Med 363: 864, 2010.

5. Schwab JH, Boland P, Guo T, et al. Skeletal metastases in myxoid liposarcoma: an unusual pattern of distant spread. Ann Surg Oncol 14: 1507-1514, 2007.

6. Thway K, Flora R, Shah C, Olmos D, Fisher C. Diagnostic utility of p16, CDK4, and MDM2 as an immunohistochemical panel in distinguishing well-differentiated and dedifferentiated liposarcomas from other adipocytic tumors. Am J Surg Pathol 36: 462-469, 2012.

7. Cahlon O, Brennan MF, Jia X, Qin LX, Singer S, Alektiar KM. A postoperative nomogram for local recurrence risk in extremity soft tissue sarcomas after limb-sparing surgery without adjuvant radiation. Ann Surg 255: 343-347, 2012.

8. Hogg ME, Wayne JD. Atypical lipomatous tumor/well-differentiated liposarcoma: what is it? Surg Oncol Clin N Am 21: 333340, 2012.

9. Dei Tos AP, Doglioni C, Piccinin S, et al. Coordinated expression and amplification of the MDM2, CDK4, and HMGI-C genes in atypical lipomatous tumours. J Pathol 190: 531-536, 2000.

10. Hostein I, Pelmus M, Aurias A, Pedeutour F, Mathoulin-Pélissier $\mathrm{S}$, Coindre JM. Evaluation of MDM2 and CDK4 amplification by real-time PCR on paraffin wax-embedded material: a potential tool for the diagnosis of atypical lipomatous tumours/well-differentiated liposarcomas. J Pathol 202: 95-102, 2004.

11. Pilotti S, Della Torre G, Mezzelani A, et al. The expression of MDM2/CDK4 gene product in the differential diagnosis of well differentiated liposarcoma and large deep-seated lipoma. Br J Cancer 82: 1271-1275, 2000.

12. Crago AM, Socci ND, DeCarolis P, et al. Copy number losses define subgroups of dedifferentiated liposarcoma with poor prognosis and genomic instability. Clin Cancer Res 18: 1334-1340, 2012.

13. Mussi C, Colombo $P$, Bertuzzi A, et al. Retroperitoneal sarcoma: is it time to change the surgical policy? Ann Surg Oncol 18: 2136-2142, 2011.

14. Sekino Y, Kobayashi A, Takagi S, Miwa S, Miyagawa S. Successful treatment for combined pancreatoduodenal injury by a secondstage pancreatojejunostomy following pancreatoduodenectomy. Hepatogastroenterology 51: 1674-1675, 2004.

15. Miyagawa S, Makuuchi M, Kawasaki S, Ogiwara M. Secondstage pancreatojejunostomy following pancreatoduodenectomy in 
high-risk patients. Am J Surg 168: 66-68, 1994.

16. Maeda H, Okabayashi T, Kobayashi M, et al. Emergency pancreatoduodenectomy for pancreatic metastasis from renal cell carcinoma in a patient with von Hippel-Lindau disease: a case report. Dig Dis Sci 51: 1383-1387, 2006.

17. Uzunoglu FG, Stehr A, Fink JA, et al. Ultrasonic dissection versus conventional dissection techniques in pancreatic surgery: a randomized multicentre study. Ann Surg 256: 675-679, 2012.

18. Ahmad SA, Edwards MJ, Sutton JM, et al. Factors influencing readmission after pancreaticoduodenectomy: a multi-institutional study of 1302 patients. Ann Surg 256: 529-537, 2012.

19. Braga M, Capretti G, Pecorelli N, et al. A prognostic score to pre- dict major complications after pancreaticoduodenectomy. Ann Surg 254: 702-707, 2011.

20. Tan AY, Riley TR, Coady T, Bussemaker HJ, Manley JL. TLS/ FUS (translocated in liposarcoma/fused in sarcoma) regulates target gene transcription via single-stranded DNA response elements. Proc Natl Acad Sci U S A 109: 6030-6035, 2012.

21. Hunder NN, Wallen H, Cao J, et al. Treatment of metastatic melanoma with autologous CD4+ T cells against NY-ESO-1. N Engl J Med 358: 2698-2703, 2008.

22. Pollack SM, Jungbluth AA, Hoch BL, et al. NY-ESO-1 is a ubiquitous immunotherapeutic target antigen for patients with myxoid/round cell liposarcoma. Cancer 118: 4564-4570, 2012.

(C) 2013 The Japanese Society of Internal Medicine http://www.naika.or.jp/imonline/index.html 